\title{
THE NUMBER OF BOUNDARY CONDITIONS FOR INITIAL BOUNDARY VALUE PROBLEMS*
}

\author{
JAN NORDSTRÖM ${ }^{\dagger}$ AND THOMAS M. HAGSTROM ${ }^{\ddagger}$
}

\begin{abstract}
Both the energy method and the Laplace transform method are frequently used for determining the number of boundary conditions required for a well posed initial boundary value problem. We show that these two distinctly different methods yield the same results. The continuous energy method can be mimicked exactly in the corresponding semidiscrete problems discretized using the summation-by-parts technique. Hence the analysis of well posedness and stability can bypass the more unwieldy Laplace transform method.
\end{abstract}

Key words. initial boundary value problems, boundary conditions, incompletely parabolic, energy method, Laplace transform method, normal mode analysis, summation-by-parts

AMS subject classifications. $35 \mathrm{Mxx}, 65 \mathrm{Mxx}$

DOI. $10.1137 / 20 \mathrm{M} 1322571$

1. Introduction. It is common to employ either the energy method or the Laplace transform method in order to determine how many boundary conditions an initial boundary value problem (IBVP) requires for well posedness. The energy method builds on integration-by-parts (see Kreiss and Lorenz (1989), Gustafsson, Kreiss, and Oliger (1995), Gustafsson and Sundström (1978), Nordström and Svärd (2005), Nordström (2017)). One obtains an expression for the energy rate that involves boundary terms. The number of boundary conditions is given by the minimal number that limits these boundary terms.

The Laplace transform method is different and employs an expansion of the solution in modes (see Hersh (1963), Hersh (1964), Kreiss (1970), Strikwerda (1977), Sakamoto (1982), Engquist and Gustafsson (1987), Eriksson and Nordström (2017)) that turns the IBVP into a one-dimensional boundary value problem. The number of boundary conditions is given by the number of conditions required to determine these modes. These two methods give the same result on well-known equation sets (see Nordström (1989), Nordström and Gustafsson (2003), Nordström, Mattsson, and Swanson (2007), Lauren and Nordström (2018)). In this paper we explain why.

Since the Laplace transform method provides necessary and sufficient conditions for well posedness (Kreiss (1970), Strikwerda (1977)) of IBVPs, this implies that the more easy-to-apply energy method will as well — at least for hyperbolic-parabolic systems. The energy method for the continuous problem can be mimicked exactly in the corresponding semidiscrete problem if discretized using the summation-by-parts (SBP) technique (Kreiss and Scherer $(1974,1977)$ ) augmented with weak boundary

* Received by the editors March 2, 2020; accepted for publication (in revised form) July 21, 2020; published electronically October 7, 2020. Any opinions, findings, and conclusions or recommendations expressed in this material are those of the authors and do not necessarily reflect the views of the National Science Foundation or the views of Vetenskapsrådet.

https://doi.org/10.1137/20M1322571

Funding: The first author was supported by Vetenskapsrådet, Sweden grant 2018-05084 VR. The second author was supported by National Science Foundation grant DMS-2012296.

${ }^{\dagger}$ Department of Mathematics, Linköping University, SE-581 83 Linköping, Sweden, and Department of Mathematics and Applied Mathematics, University of Johannesburg, Auckland Park 2006, Johannesburg, South Africa (jan.nordstrom@liu.se).

${ }^{\ddagger}$ Department of Mathematics, Southern Methodist University, Dallas, TX 75275-0156 USA (thagstrom@mail.smu.edu).

2818 
conditions (simultaneous-approximation-term (SAT) technique (Carpenter, Gottlieb, and Abarbanel (1994))). We will discuss what our results indicate for semidiscrete approximations in SBP-SAT form, such as finite differences (Nordström et al. (2009); Svärd, Carpenter, and Nordström (2007); Svärd and Nordström (2008)), finite volume (Nordström, Eriksson, and Eliasson (2012); Nordström et al. (2003)), spectral elements (Carpenter et al. (2014); Carpenter and Gottlieb (1996); Yan et al. (2020)), flux reconstruction (Castonguay et al. (2013); Huynh (2007); Ranocha, Öffner, and Sonar (2016)) and discontinuous Galerkin schemes (Kopriva and Gassner (2014); Gassner (2013); Hesthaven and Gottlieb (1996); Bassi and Rebay (1997); Hartmann and Houston (2008)). For reviews, see Svärd and Nordström (2014) and Del Rey Fernandez, Hicken, and Zingg (2014).

2. Preliminaries. We start by presenting the general system of equations that will be investigated, and we provide basic results for the Cauchy problem.

2.1. The system of partial differential equations. The three-dimensional constant coefficient system of equations that we will consider is

$$
V_{t}+\bar{A} V_{x}+\bar{B} V_{y}+\bar{C} V_{z}=\left(\bar{D}_{11} V_{x}+\bar{D}_{12} V_{y}+\bar{D}_{13} V_{z}\right)_{x}+\left(\bar{D}_{21} V_{x}+\bar{D}_{22} V_{y}+\bar{D}_{23} V_{z}\right)_{y}
$$

$$
+\left(\bar{D}_{31} V_{x}+\bar{D}_{32} V_{y}+\bar{D}_{33} V_{z}\right)_{z}
$$

In (2.1), $V$ is the solution, $\bar{A}, \bar{B}, \bar{C}$ are symmetric matrices related to the hyperbolic terms, and the parabolic matrices $\bar{D}_{11}, \bar{D}_{22}, \bar{D}_{33}, \bar{D}_{12}+\bar{D}_{21}, \bar{D}_{13}+\bar{D}_{31}, \bar{D}_{23}+\bar{D}_{32}$ are symmetric. In the incompletely parabolic case, the structure of the matrices $\bar{D}_{i j}$ in block form is

$$
\bar{D}_{i j}=\epsilon\left(\begin{array}{cc}
\left(D_{i j}\right)_{m \times m} & 0_{m \times n} \\
0_{n \times m} & 0_{n \times n}
\end{array}\right)
$$

where subscripts indicate the size of the matrices, and $\epsilon>0$ is a constant parameter. In the parabolic case, the matrices $\bar{D}_{i j}$ are full. For $\epsilon \equiv 0$, the problem is symmetric hyperbolic and hence well posed.

Under the assumptions on the matrices $D_{i j}$ listed in (2.5) below, (2.1) is a well posed parabolic or incompletely parabolic system also for $\epsilon>0$. In most of the paper we focus on the incompletely parabolic case, and we will comment shortly on the difference between that and the hyperbolic or parabolic cases. We note that there has also been related work dealing with the presence or suppression of boundary layers in the case $\epsilon \ll 1$ (e.g., Michelson (1985); Halpern (1991); Lohéac (1991)), but we do not consider that issue here.

REMARK 1. Abarbanel and Gottlieb (1981) showed that the three-dimensional compressible Navier-Stokes equations with 12 matrices of the form (2.1) can be symmetrized by a single matrix (symmetrizer). In addition, they observed that at least two different symmetrizers exist, based on either the hyperbolic or the parabolic terms.

2.2. The Cauchy and half space problem. The Cauchy problem for (2.1) can be Fourier transformed in all coordinates, which yields

$$
\begin{aligned}
& \hat{V}_{t}+i\left(\omega_{x} \bar{A}+\omega_{y} \bar{B}+\omega_{z} \bar{C}\right) \hat{V}+\left[\omega_{x}^{2} \bar{D}_{11}+\omega_{y}^{2} \bar{D}_{22}+\omega_{z}^{2} \bar{D}_{33}\right. \\
& \left.\quad+\omega_{x} \omega_{y}\left(\bar{D}_{12}+\bar{D}_{21}\right)+\omega_{y} \omega_{z}\left(\bar{D}_{23}+\bar{D}_{32}\right)+\omega_{z} \omega_{x}\left(\bar{D}_{13}+\bar{D}_{31}\right)\right] \hat{V}=0
\end{aligned}
$$


where $\omega_{x}, \omega_{y}$, and $\omega_{z}$ are the wave numbers in the $x, y$, and $z$ directions, respectively. By multiplying (2.3) with $\hat{V}^{*}$ (the complex conjugated $\hat{V}$ ) from the left, we get

$$
|\hat{V}|_{t}^{2}+2\left(\begin{array}{c}
i \omega_{x} \hat{V} \\
i \omega_{y} \hat{V} \\
i \omega_{z} \hat{V}
\end{array}\right)^{*} \underbrace{\left(\begin{array}{ccc}
\bar{D}_{11} & \bar{D}_{12} & \bar{D}_{13} \\
\bar{D}_{21} & \bar{D}_{22} & \bar{D}_{23} \\
\bar{D}_{31} & \bar{D}_{32} & \bar{D}_{33}
\end{array}\right)}_{\bar{D}}\left(\begin{array}{c}
i \omega_{x} \hat{V} \\
i \omega_{y} \hat{V} \\
i \omega_{z} \hat{V}
\end{array}\right)=0 .
$$

For $\epsilon>0$, we demand that the second order terms are Petrovskii parabolic, i.e.,

$$
\begin{aligned}
& \bar{D} \geq 0, D_{11}>0, D_{22}>0, D_{33}>0, \\
& \omega_{x}^{2} D_{11}+\omega_{y}^{2} D_{22}+\omega_{x} \omega_{y}\left(D_{12}+D_{21}\right)>0, \\
& \omega_{y}^{2} D_{22}+\omega_{z}^{2} D_{33}+\omega_{y} \omega_{z}\left(D_{23}+D_{32}\right)>0, \\
& \omega_{z}^{2} D_{33}+\omega_{x}^{2} D_{11}+\omega_{z} \omega_{x}\left(D_{13}+D_{31}\right)>0,
\end{aligned}
$$

for values of $\omega_{x}, \omega_{y}, \omega_{z}$ not vanishing simultaneously. Note that $\bar{D}$ is positive definite in the parabolic case and positive semidefinite in the incompletely parabolic case. With conditions (2.5), the Cauchy problem (2.3) is well posed.

The upcoming analysis will be performed in the half space $\Omega:\{x>0,-\infty<$ $y, z<+\infty\}$, with boundary conditions imposed at the plane $x=0$. The Fourier transformed equations in $y, z$ stemming from (2.1) that we will analyze read

$$
\begin{aligned}
\hat{V}_{t} & +\left[\bar{A}-i \omega_{y}\left(\bar{D}_{12}+\bar{D}_{21}\right)-i \omega_{z}\left(\bar{D}_{13}+\bar{D}_{31}\right)\right] \hat{V}_{x}-\bar{D}_{11} \hat{V}_{x x} \\
& +\left[i\left(\omega_{y} \bar{B}+\omega_{z} \bar{C}\right)+\omega_{y}^{2} \bar{D}_{22}+\omega_{z}^{2} \bar{D}_{33}+\omega_{y} \omega_{z}\left(\bar{D}_{23}+\bar{D}_{32}\right)\right] \hat{V}=0 .
\end{aligned}
$$

3. Analysis. Our ambition in this paper is to prove that the energy method and the Laplace transform method lead to the same number of boundary conditions for the systems (2.1) and (2.6).

3.1. The Laplace transform analysis. To determine the number of boundary conditions, the ansatz $\hat{V}=\psi e^{\kappa x+s t}$, with $\kappa=\kappa^{R}+i \omega_{x}$ and $s=\eta+i \xi$, is inserted into (2.6). We find

$$
\begin{aligned}
(s I & +\left[\bar{A}-i \omega_{y}\left(\bar{D}_{12}+\bar{D}_{21}\right)-i \omega_{z}\left(\bar{D}_{13}+\bar{D}_{31}\right)\right] \kappa-\bar{D}_{11} \kappa^{2} \\
& \left.+\left[i\left(\omega_{y} \bar{B}+\omega_{z} \bar{C}\right) \hat{V}+\omega_{y}^{2} \bar{D}_{22}+\omega_{z}^{2} \bar{D}_{33}+\omega_{y} \omega_{z}\left(\bar{D}_{23}+\bar{D}_{32}\right)\right]\right) \psi=0 .
\end{aligned}
$$

The number of boundary conditions at $x=0$ is given by the number of modes related to $\operatorname{Re}(\kappa)=\kappa^{R}<0$ for $\operatorname{Re}(s)=\eta>0$. These modes decay away from the boundary $x=0$, and the roots $\kappa$ are obtained from

$$
\begin{aligned}
\operatorname{Det}(s I & +\left[\bar{A}-i \omega_{y}\left(\bar{D}_{12}+\bar{D}_{21}\right)-i \omega_{z}\left(\bar{D}_{13}+\bar{D}_{31}\right)\right] \kappa-\bar{D}_{11} \kappa^{2} \\
& \left.+i\left(\omega_{y} \bar{B}+\omega_{z} \bar{C}\right) \hat{V}+\omega_{y}^{2} \bar{D}_{22}+\omega_{z}^{2} \bar{D}_{33}+\omega_{y} \omega_{z}\left(\bar{D}_{23}+\bar{D}_{32}\right)\right)=0 .
\end{aligned}
$$

To solve the problem (3.2) for all roots $\kappa$ poses a significant challenge. (For example, for the three-dimensional Navier-Stokes equations, (3.2) is a 9th degree polynomial in $\kappa$.)

Luckily, there are simplifying circumstances. The following lemma will come in handy.

LEMma 1. The number of roots, $\kappa$, with positive and negative real parts is independent of $s$ and the wave numbers $\omega_{y}, \omega_{z}$ for $\Re(s)>0$.

Copyright $@$ by SIAM. Unauthorized reproduction of this article is prohibited. 
Proof. First, we show that there are no imaginary roots $\kappa=i \omega_{x}$ for $\operatorname{Re}(s)>0$. By inserting $\kappa=i \omega_{x}$ into (3.1), multiplying from the left with $\psi^{*}$, and adding the transpose, we find

$$
\begin{aligned}
\psi^{*}(\eta I & +\omega_{x}^{2} \bar{D}_{11}+\omega_{y}^{2} \bar{D}_{22}+\omega_{z}^{2} \bar{D}_{33} \\
& \left.+\omega_{x} \omega_{y}\left(\bar{D}_{12}+\bar{D}_{21}\right)+\omega_{y} \omega_{z}\left(\bar{D}_{23}+\bar{D}_{32}\right)+\omega_{z} \omega_{x}\left(\bar{D}_{13}+\bar{D}_{31}\right)\right) \psi=0
\end{aligned}
$$

By (2.5) we can conclude that $\psi=0$ for $\operatorname{Re}(s)=\eta>0$ for both the hyperbolicparabolic and incompletely parabolic cases.

Second, to complete the proof we need all roots $\kappa$ to be bounded for bounded $\left(s, \omega_{y}, \omega_{z}\right)$. Then, for $\left(s, \omega_{y}, \omega_{z}\right)$ restricted to any bounded region $\mathcal{R}$, we can find a closed contour $\mathcal{C}$ bounded by the imaginary axis and a curve in $\operatorname{Re}(\kappa) \geq 0$ such that no roots can ever intersect $\mathcal{C}$ as $\left(s, \omega_{y}, \omega_{z}\right)$ varies in $\mathcal{R}$. The fact that the number of roots (counting multiplicities) within $\mathcal{C}$ is the same for all points in $\mathcal{R}$ follows from the continuity of the logarithmic derivative of the characteristic polynomial on $\mathcal{C}$ and an application of the argument principle Marden (1949, Chap. 1).

Third and last, the boundedness of $\kappa$ follows immediately from the nonsingular $\bar{D}_{11}$ in the parabolic case. In the more complex incompletely parabolic case, the boundedness of $\kappa$ is established by a simple perturbation argument in Lemma 5, whose formulation and proof we defer to the appendix. A refinement of the argument given there also applies in the hyperbolic case.

REMARK 2. Lemma 1 simplifies the analysis since we can set $\omega_{y}=\omega_{z}=0$ and choose any $s$ with $\operatorname{Re}(s)>0$ to determine the number of roots $\kappa$ with positive and negative real parts. We can, for example, choose $s$ with $\operatorname{Re}(s) \rightarrow \infty$, which often simplifies the algebra.

Due to Lemma 1, it is sufficient to consider the one-dimensional problem when determining the number of boundary conditions at $x=0$. The number of roots with $\operatorname{Re}(\kappa)=\kappa^{R}<0$ for $\operatorname{Re}(s)>0$ is thus obtained from

$$
\operatorname{Det}\left(s I+\bar{A} \kappa-\bar{D}_{11} \kappa^{2}\right)=0 .
$$

REMARK 3. The discrete version of the Laplace transform technique (also denoted normal mode analysis) is more complicated and less applicable than the continuous version. The resulting number of discrete modes is the sum of necessary boundary conditions and numerical boundary closures (Gustafsson, Kreiss, and Sundström (1972); Coulombel $(2009,2011))$. The applicability of the normal mode analysis is restricted to equidistant meshes, which essentially limits it to finite difference methods. Even for finite difference methods, the discrete analysis becomes very involved at high orders of accuracy and must be redone for each order.

3.2. The energy analysis. In the energy analysis, (2.6) is multiplied with the solution and integrated from zero to infinity. We find (see Nordström (2017))

$$
\|\hat{V}\|_{t}^{2}+2 \int_{0}^{\infty}\left(\begin{array}{c}
\hat{V}_{x} \\
i \omega_{y} \hat{V} \\
i \omega_{z} \hat{V}
\end{array}\right)^{*}\left(\begin{array}{ccc}
\bar{D}_{11} & \bar{D}_{12} & \bar{D}_{13} \\
\bar{D}_{21} & \bar{D}_{22} & \bar{D}_{23} \\
\bar{D}_{31} & \bar{D}_{32} & \bar{D}_{33}
\end{array}\right)\left(\begin{array}{c}
\hat{V}_{x} \\
i \omega_{y} \hat{V} \\
i \omega_{z} \hat{V}
\end{array}\right) d x=B T
$$

where

$$
B T=\left(\begin{array}{c}
\hat{V} \\
\hat{V}_{x}
\end{array}\right)^{*}\left(\begin{array}{cc}
\bar{A} & -\bar{D}_{11} \\
-\bar{D}_{11} & 0
\end{array}\right)\left(\begin{array}{c}
\hat{V} \\
\hat{V}_{x}
\end{array}\right)
$$

Copyright (c) by SIAM. Unauthorized reproduction of this article is prohibited. 
In (3.5), the boundary term $B T$ is evaluated at $x=0$, and the values at $\infty$ are ignored.

The total number of boundary conditions in the energy method is given by the number of positive eigenvalues $\lambda$ in

$$
\operatorname{Det}\left(\left(\begin{array}{cc}
\bar{A} & -\bar{D}_{11} \\
-\bar{D}_{11} & 0
\end{array}\right)-\left(\begin{array}{cc}
\lambda I & 0 \\
0 & \lambda I
\end{array}\right)\right)=0
$$

REMARK 4. The total number of positive and negative eigenvalues can be calculated by invoking Lemma 4 below and computing a representation of the matrix in the form $R^{T} D R$ where $D$ is diagonal; see, e.g., Golub and van Loan (1989, Chap. 4).

3.3. The relation between the Laplace and energy methods. The question that we will discuss in this paper arises by comparing (3.3) for $\kappa$ and (3.6) for $\lambda$. In the hyperbolic case, where $\bar{D}_{11}=0$, the two equations are similar. By comparison we find that $\kappa=-s / \lambda$, and hence the two formulations yield exactly the same number of boundary conditions.

In the parabolic/incompletely parabolic case, the two equations have similar ingredients (the matrices $\bar{A}$ and $\bar{D}_{11}$ ) but are otherwise quite different. However, to the best of our knowledge, these two methods always provide the same number of boundary conditions. The question is, How can that be possible?

To answer that question, let us return to the governing system of equations (2.6) with the simplifying insertion of $\omega_{y}=\omega_{z}=0$, making the problem one-dimensional:

$$
U_{t}+\bar{A} U_{x}-\bar{D}_{11} U_{x x}=0 \text {. }
$$

To ease notation, we replaced $\hat{V}$ with $U$ in (3.7). The matrix structure in (2.2) for the incompletely parabolic case suggests the block form

$$
U=\left(\begin{array}{c}
U_{1} \\
U_{2}
\end{array}\right), \quad \bar{A}=\left(\begin{array}{ll}
A_{11} & A_{12} \\
A_{21} & A_{22}
\end{array}\right), \quad \bar{D}_{11}=\left(\begin{array}{cc}
D_{11} & 0 \\
0 & 0
\end{array}\right) .
$$

REMARK 5. We perform the analysis for the more complicated incompletely parabolic case as indicated by (3.8). The derivation in the parabolic case is similar but easier.

REMARK 6. The simplification to a purely one-dimensional study is possible due to Lemma 1 in combination with conditions (3.3) and (3.6).

Next we transform (3.7) to first order form by introducing $\phi=\left(U_{1}\right)_{x}$. The new set of governing equations becomes

$$
\left[\begin{array}{lll}
I & 0 & 0 \\
0 & I & 0 \\
0 & 0 & 0
\end{array}\right]\left[\begin{array}{c}
U_{1} \\
U_{2} \\
\phi
\end{array}\right]_{t}+\left[\begin{array}{ccc}
A_{11} & A_{12} & -D_{11} \\
A_{21} & A_{22} & 0 \\
-D_{11} & 0 & 0
\end{array}\right]\left[\begin{array}{c}
U_{1} \\
U_{2} \\
\phi
\end{array}\right]_{x}+\left[\begin{array}{ccc}
0 & 0 & 0 \\
0 & 0 & 0 \\
0 & 0 & D_{11}
\end{array}\right]\left[\begin{array}{c}
U_{1} \\
U_{2} \\
\phi
\end{array}\right]=0 .
$$

The energy method applied to (3.9) yields the energy rate

$$
\|U\|_{t}^{2}+2 \int_{0}^{\infty} \phi^{T} D_{11} \phi d x=B T,
$$

where

$$
B T=\left(\begin{array}{c}
U_{1} \\
U_{2} \\
\phi
\end{array}\right)^{T} \underbrace{\left(\begin{array}{ccc}
A_{11} & A_{12} & -D_{11} \\
A_{21} & A_{22} & 0 \\
-D_{11} & 0 & 0
\end{array}\right)}_{E}\left(\begin{array}{c}
U_{1} \\
U_{2} \\
\phi
\end{array}\right) .
$$

Copyright (c) by SIAM. Unauthorized reproduction of this article is prohibited. 
The boundary term $B T$ is evaluated at $x=0$. The total number of boundary conditions at $x=0$ is given by the number of positive eigenvalues $\lambda$ satisfying

$$
\operatorname{Det}(E-\lambda I)=0 \text {. }
$$

To formalize the result above we state the following lemma.

Lemma 2. The eigenvalues satisfying (3.12) are identical to the nonzero eigenvalues in (3.6).

Proof. From (3.8), (3.11), and (3.12), the matrix $E$ is the same as the matrix in (3.6) after removing the zero rows and columns.

The Laplace transform method applied to (3.9) with the ansatz $\left(U_{1}, U_{2}, \phi\right)^{T}=$ $\psi e^{\kappa x+s t}$ yields the new generalized eigenvalue problem,

$$
(\underbrace{\left(\begin{array}{ccc}
A_{11} & A_{12} & -D_{11} \\
A_{21} & A_{22} & 0 \\
-D_{11} & 0 & 0
\end{array}\right)}_{E} \kappa+\left[\begin{array}{ccc}
s I & 0 & 0 \\
0 & s I & 0 \\
0 & 0 & D_{11}
\end{array}\right]) \psi=0,
$$

where the matrix $E$ in the energy rate (3.11) shows up again. The roots $\kappa$ are given by

$$
\operatorname{Det}\left(E \kappa+\left[\begin{array}{ccc}
s I & 0 & 0 \\
0 & s I & 0 \\
0 & 0 & D_{11}
\end{array}\right]\right)=0 .
$$

To guarantee uniqueness, we need the next lemma.

Lemma 3. The determinant condition (3.14) leads to the same roots $\kappa$ as (3.3).

Proof. Consider (3.14) and recall that adding a row (column) multiplied by a scalar to another row (column) does not change the determinant. By adding $\kappa$ times the lower block rows to the upper block rows, we find

$$
\begin{aligned}
& \operatorname{Det}\left(\begin{array}{ccc}
A_{11} \kappa+s I-D_{11} \kappa^{2} & A_{12} \kappa & 0 \\
A_{21} \kappa & A_{22} \kappa+s I & 0 \\
-D_{11} \kappa & 0 & D_{11}
\end{array}\right) \\
& =\operatorname{Det}\left(\begin{array}{cc}
A_{11} \kappa+s I-D_{11} \kappa^{2} & A_{12} \kappa \\
A_{21} \kappa & A_{22} \kappa+s I
\end{array}\right) \times \operatorname{Det}\left(D_{11}\right)=0 .
\end{aligned}
$$

Since $\operatorname{Det}\left(D_{11}\right) \neq 0$, inserting the relations (3.8) into (3.3) proves the claim.

REMARK 7. The matrix $E$ is the link between the energy and Laplace transform methods. It connects the roots $\kappa$ in (3.3) from the Laplace transform method to the eigenvalues $\lambda$ in (3.6) from the energy method.

To proceed, we also need the following lemma from Nordström and Svärd (2005).

Lemma 4. Suppose that $R$ is a nonsingular matrix and that $P$ is a real symmetric matrix. Then the number of positive/negative eigenvalues of $R^{T} P R$ is the same as the number of positive/negative eigenvalues of $P$.

Proof. The proof follows directly from Sylvester's criterion; see Horn and Johnson (1990).

Copyright (c) by SIAM. Unauthorized reproduction of this article is prohibited. 
We can now prove the main result of this paper.

THEOREM 1. Consider the system of partial differential equations (2.1) posed on the domain $\Omega:\{x>0,-\infty<y, z<+\infty\}$ with the boundary conditions imposed at the plane $x=0$. The energy and Laplace transform methods applied to that half plane problem lead to the same number of boundary conditions at $x=0$.

The proof for the hyperbolic case is trivial and given in the first paragraph of this subsection. Below, we give the proof for the incompletely parabolic case. The parabolic case can be treated similarly, with more ease.

Proof. We will show that (i) the total number of nonzero eigenvalues $\lambda$ in (3.12) and the total number of nonzero roots $\kappa$ in (3.14) for $R e(s)>0$ are identical, and (ii) the number of positive $\lambda$ in (3.12) is the same as the number of roots $\kappa$ with $\operatorname{Re}(\kappa)<0$ for $\operatorname{Re}(s)>0$. If both (i) and (ii) hold, Theorem 1 follows since Lemmas 2 and 3 show the equivalence between the first and second order forms.

Consider the matrix in (3.14). By Lemma 1 we can choose any $s$ with $\operatorname{Re}(s)>0$ and get the correct number of roots $\kappa$ with negative real part. We choose the specific value $s=1$. Since the matrix $D_{11}$ is symmetric positive definite, we can factorize it and get

$$
\left(\begin{array}{ccc}
s I & 0 & 0 \\
0 & s I & 0 \\
0 & 0 & D_{11}
\end{array}\right) \rightarrow(s=1) \rightarrow\left(\begin{array}{ccc}
I & 0 & 0 \\
0 & I & 0 \\
0 & 0 & D_{11}^{1 / 2}
\end{array}\right)\left(\begin{array}{ccc}
I & 0 & 0 \\
0 & I & 0 \\
0 & 0 & D_{11}^{1 / 2}
\end{array}\right)=S S
$$

Now we can insert these matrices into (3.14), noting that $S$ is symmetric, and find

$$
\operatorname{Det}\left(S\left(S^{-1} E S^{-1}+\kappa^{-1} I\right) S\right)=\operatorname{Det}(S)^{2} \operatorname{Det}\left(\left(S^{-1}\right)^{T} E S^{-1}+\kappa^{-1} I\right) .
$$

The final result for (i) above follows from Lemma 4. Also (ii) follows since the number of roots with $\operatorname{Re}(\kappa)<0$ is the same as the number of positive eigenvalues $\lambda$ of $E$.

3.4. The energy method and discretization schemes. The energy method (in contrast to the Laplace transform method; see Remark 3) is method agnostic for semidiscrete problems in SBP-SAT or Galerkin form. It does not require additional numerical boundary closures (they are already included in the SBP operators) and is applicable to both nonuniform meshes and different orders of accuracy. Practically, energy-stable boundary closures can be obtained by penalty formulations in either the SBP-SAT or Galerkin framework. For methods which do not introduce new variables, such as SBP-based difference methods, continuous Galerkin, or interiorpenalty-based discontinuous Galerkin methods, one obtains semidiscretizations of the half space problem (2.1) in $x>0$ which, after integration or SBP, have the form

$$
\left\langle\Phi, V_{t}\right\rangle+\langle\Phi, L V\rangle-\langle\Phi, B V\rangle_{x=0}+\langle P \Phi, S V\rangle_{x=0}=0
$$

where $\langle$,$\rangle is an inner product, \Phi$ is an arbitrary test function or grid function, $L+L^{T} \geq 0$ (here transpose is understood in terms of the inner product), $B$ is a boundary operator arising from the integration of SBP procedure, and $P, S$ are penalty operators used to weakly impose the boundary condition. In particular,

$$
B V \approx-\left(\bar{D}_{11} V_{x}+\bar{D}_{12} V_{y}+\bar{D}_{13} V_{z}\right)+\frac{1}{2} \bar{A} V \equiv-\bar{D}_{11} F_{x}+\frac{1}{2} \bar{A} V,
$$

where we have factored out $\bar{D}_{11}$ from $F$. 
We now assume that the boundary condition imposed at $x=0$ is dissipative or neutral (such a boundary condition is always possible to impose for hyperbolicparabolic systems). On the continuous level this means that for functions satisfying the boundary condition

$$
H\left(\begin{array}{c}
V \\
F_{x}
\end{array}\right)=0
$$

we have

$$
-V^{T} \bar{D}_{11} F_{x}+\frac{1}{2} V^{T} A V=\frac{1}{2}\left(\begin{array}{c}
V \\
F_{x}
\end{array}\right)^{T}\left(\begin{array}{cc}
\bar{A} & -\bar{D}_{11} \\
-\bar{D}_{11} & 0
\end{array}\right)\left(\begin{array}{c}
V \\
F_{x}
\end{array}\right) \geq 0,
$$

where again we see the matrix $E$ appearing in (3.6) and (3.12).

To show that (3.20) holds with (3.19) imposed, we start by diagonalizing $E$,

$$
\frac{1}{2}\left(\begin{array}{cc}
\bar{A} & -\bar{D}_{11} \\
-\bar{D}_{11} & 0
\end{array}\right)=Q\left(\begin{array}{cc}
\Lambda^{+} & 0 \\
0 & \Lambda^{-}
\end{array}\right) Q^{T}, \Lambda^{+}>0, \Lambda^{-} \leq 0,
$$

and set

$$
Q^{T}\left(\begin{array}{c}
V \\
F_{x}
\end{array}\right)=\left(\begin{array}{c}
W^{+} \\
W^{-}
\end{array}\right)
$$

Then a boundary matrix $H$ of rank equal to the dimensionality of $\Lambda^{+}$leading to energy stability must be of the form $H=\left(\begin{array}{ll}I & -R\end{array}\right) Q^{T}$, which transforms (3.19) into $W^{+}-R W^{-}=0$. It can be shown (see Nordström (2017)) that an energy estimate using strong boundary conditions requires a matrix $R$ that satisfies

$$
\Lambda^{-}+R^{T} \Lambda^{+} R \leq 0 .
$$

For the semidiscretized system (3.17) we must specify the penalty terms. Note that the construction of $H$ is algebraic and applies to the approximate derivatives appearing in $B$ as well as to the soon-to-be-chosen operators $S$ and $P$. In the equations below, the quantities $W^{ \pm}$will be understood to satisfy (3.22), with the continuous quantities replaced by approximate ones.

To be consistent with the continuous formulation, we first choose $S=H$. Then if we choose

$$
P=\left(\Lambda^{+} \Lambda^{+} R\right) Q^{T},
$$

the combined boundary contribution to the energy for the semidiscrete problem becomes

$$
\begin{aligned}
(P V)^{T} S V-V^{T} B V= & \left(\Lambda^{+}\left(W^{+}+R W^{-}\right)\right)^{T}\left(W^{+}-R W^{-}\right) \\
& -\left(W^{+}\right)^{T} \Lambda^{+} W^{+}-\left(W^{-}\right)^{T} \Lambda^{-} W^{-} \\
= & -\left(W^{-}\right)^{T}\left(\Lambda^{-}+R^{T} \Lambda^{+} R\right) W^{-} \geq 0 .
\end{aligned}
$$

Thus by the requirement (3.23), the method is energy stable.

The construction above is applicable to a wide array of methods, such as continuous Galerkin finite elements, interior-penalty discontinuous Galerkin finite elements, and SBP finite differences. With minor changes associated with the additional flux variables, it can also be applied to local discontinuous Galerkin methods. We note that other penalizations may also be used, but ours is a simple and universal choice. 
4. Conclusions. We have investigated the energy method and the Laplace transform method as techniques for determining the number of boundary conditions required for a well posed IBVP. By reducing the systems of equations to first order form, we proved that both methods yield the same number of boundary conditions.

The Laplace transform method is known to provide necessary and sufficient conditions for the well posedness of IBVPs, and our result shows that the energy method will provide them as well for hyperbolic, parabolic, and incompletely parabolic systems. Also, since both methods can be applied pointwise at smooth boundaries, the result extends to this case.

If the IBVP is discretized using the SBP technique with weak boundary conditions, the result is directly applicable to the corresponding semidiscrete approximation. Thus, the energy method provides an easy-to-apply general approach for implementation of boundary conditions in both the continuous and semidiscrete cases. The Laplace transform method can often be bypassed.

\section{Appendix.}

Lemma 5. For bounded $\left(s, \omega_{y}, \omega_{z}\right)$ and $\operatorname{Re}(s)=\eta>0$, the roots $\kappa$ of (3.2) are bounded.

Proof. Rewrite the eigenvalue problem (3.1) in block form according to the dimensions of $D_{11}$ and recall that $\bar{A}, \bar{B}$, and $\bar{C}$ are symmetric:

$$
\begin{array}{r}
\left(\hat{P}_{11}+\hat{Q}_{11} \kappa+s I-D_{11} \kappa^{2}\right) \hat{V}_{1}+\left(i \omega_{y} \bar{B}_{12}+i \omega_{z} \bar{C}_{12}+\bar{A}_{12} \kappa\right) \hat{V}_{2}=0, \\
\left(i \omega_{y} \bar{B}_{12}^{T}+i \omega_{z} \bar{C}_{12}^{T}+\bar{A}_{12}^{T} \kappa\right) \hat{V}_{1}+\left(i \omega_{y} \bar{B}_{22}+i \omega_{z} \bar{C}_{22}+\bar{A}_{22} \kappa+s I\right) \hat{V}_{2}=0,
\end{array}
$$

where we have introduced

$$
\begin{aligned}
& \hat{P}_{11}=s I+i\left(\omega_{y} \bar{B}_{11}+\omega_{z} \bar{C}_{11}\right)+\omega_{y}^{2} D_{22}+\omega_{z}^{2} D_{33}+\omega_{y} \omega_{z}\left(D_{23}+D_{32}\right), \\
& \hat{Q}_{11}=\bar{A}_{11}-i \omega_{y}\left(D_{12}+D_{21}\right)-i \omega_{z}\left(D_{13}+D_{31}\right) .
\end{aligned}
$$

Now suppose that a uniformly bounded sequence of $\left(s, \omega_{y}, \omega_{z}\right), \operatorname{Re}(s)=\eta>0$ exists with $|\kappa| \rightarrow \infty$. Since $D_{11}$ is nonsingular, (5.1) then implies $\hat{V}_{1} \rightarrow 0$. This makes the largest term in (5.2) to be $\kappa \bar{A}_{22} \hat{V}_{2}$, so we must have $\bar{A}_{22} \hat{V}_{2} \rightarrow 0$. Since we are looking for nonzero solutions, such a scenario is only possible if $\bar{A}_{22}$ is singular with $\hat{V}_{2}$ approaching one of its null vectors, $v_{0}$. Returning to (5.1) and collecting the largest terms, we find

$$
-D_{11} \kappa^{2} \hat{V}_{1}+\kappa \bar{A}_{12} v_{0}+O(1)=0
$$

from which we conclude that

$$
\hat{V}_{1} \sim \kappa^{-1} D_{11}^{-1} \bar{A}_{12} v_{0} .
$$

Now set $\hat{V}_{2} \sim v_{0}+\kappa^{-1} w_{0}$, and consider $O(1)$ terms in (5.2):

$$
\bar{A}_{12}^{T} D_{11}^{-1} \bar{A}_{12} v_{0}+i \omega_{y} \bar{B}_{22} v_{0}+i \omega_{z} \bar{C}_{22} v_{0}+\bar{A}_{22} w_{0}+s v_{0}=0 .
$$

Multiplying by $v_{0}^{T}$ and taking the real part yields

$$
v_{0}^{T} \bar{A}_{12}^{T} D_{11}^{-1} \bar{A}_{12} v_{0}+\eta v_{0}^{T} v_{0}=0,
$$

where we have used the symmetry of $\bar{A}_{22}, \bar{B}_{22}$, and $\bar{C}_{22}$ and the fact that $v_{0}^{T} \bar{A}_{22} w_{0}=0$. Since $\eta>0$ and the first term in (5.7) is nonnegative, we have reached a contradiction. Thus $\kappa$ is bounded. 
Note that a simple refinement of this argument also establishes the result in the purely hyperbolic case.

\section{REFERENCES}

S. Abarbanel and D. Gottlieb (1981), Optimal time splitting for two- and three-dimensional Navier-Stokes equations with mixed derivatives, J. Comput. Phys., 41, pp. 1-33.

F. BASsi AND S. REBAY (1997), A high-order accurate discontinuous finite element method for the numerical solution of the compressible Navier-Stokes equations, J. Comput. Phys., 131, pp. 267279.

M. H. Carpenter, T. C. Fisher, E. J. Nielsen, and S. H. Frankel (2014), Entropy stable spectral collocation schemes for the Navier-Stokes equations: Discontinuous interfaces, SIAM J. Sci. Comput., 36, pp. B835-B867, https://doi.org/10.1137/130932193.

M. H. Carpenter and D. Gottlieb (1996), Spectral methods on arbitrary grids, J. Comput. Phys., 129, pp. $74-86$.

M. H. Carpenter, D. Gottlieb, And S. Abarbanel (1994), Time-stable boundary conditions for finite-difference schemes solving hyperbolic systems: Methodology and application to high-order compact schemes, J. Comput. Phys., 111, pp. 220-236.

P. Castonguay, D. Williams, P. Vincent, and A. Jameson (2013), Energy stable flux reconstruction schemes for advection-diffusion problems, Computer Methods Appl. Mech. Engrg., 267, pp. $400-417$.

J.-F. Coulombel (2009), Stability of finite difference schemes for hyperbolic initial boundary value problems, SIAM J. Numer. Anal., 47, pp. 2844-2871.

J.-F. Coulombel (2011), Stability of finite difference schemes for hyperbolic initial boundary value problems II, Ann. Sc. Norm. Super. Pisa Cl. Sci. (5), 10, pp. 37-98.

D. C. Del Rey Fernandez, J. E. Hicken, and D. W. ZingG (2014), Review of summation-byparts operators with simultaneous approximation terms for the numerical solution of partial differential equations, Comput. Fluids, 95, pp. 171-196.

B. Engquist And B. Gustafsson (1987), Steady state computations for wave propagation problems, Math. Comp., 49, pp. 39-64.

S. ERIKSSON AND J. NORDSTRÖM (2017), Exact non-reflecting boundary conditions revisited: Wellposedness and stability, Found. Comput. Math., 17, pp. 957-986.

G. J. Gassner (2013), A skew-symmetric discontinuous Galerkin spectral element discretization and its relation to SBP-SAT finite difference methods, SIAM J. Sci. Comput., 35, pp. A1233-A1253, https://doi.org/10.1137/120890144.

G. Golub And C. van Loan (1989), Matrix Computations, The Johns Hopkins University Press, Baltimore.

B. Gustafsson, H.-O. Kreiss, and J. Oliger (1995), Time Dependent Problems and Difference Methods, Academic Press, New York.

B. Gustafsson, H.-O. Kreiss, and A. Sundström (1972), Stability theory of difference approximations for mixed initial boundary value problems II, Math. Comp., 26, pp. 649-686.

B. Gustafsson And A. Sundström (1978), Incompletely parabolic problems in fluid dynamics, SIAM J. Appl. Math., 35, pp. 343-357, https://doi.org/10.1137/0135030.

L. Halpern (1991), Artificial boundary conditions for incompletely parabolic perturbations of hyperbolic systems, SIAM J. Math. Anal., 22, pp. 1256-1283, https://doi.org/10.1137/0522081.

R. Hartmann and P. Houston (2008), An optimal order interior penalty discontinuous Galerkin discretization of the compressible Navier-Stokes equations, J. Comput. Phys., 227, pp. 96709685.

R. Hersh (1963), Mixed problems in several variables, J. Math. Mech., 12, pp. 317-334.

R. Hersh (1964), Boundary conditions for equations of evolution, Arch. Ration. Mech. Anal., 16, pp. $243-264$.

J. S. Hesthaven and D. Gottlieb (1996), A stable penalty method for the compressible NavierStokes equations I: Open boundary conditions, SIAM J. Sci. Comput., 17, pp. 579-612, https: //doi.org/10.1137/S1064827594268488.

R. A. Horn And C. R. Johnson (1990), Matrix Analysis, Cambridge University Press, Cambridge, UK.

H. T. HuYnh (2007), A flux reconstruction approach to high-order schemes including discontinuous Galerkin methods, in Proceedings of the 18th AIAA Computational Fluid Dynamics Conference, AIAA-2007-4079.

Copyright $@$ ( ) by SIAM. Unauthorized reproduction of this article is prohibited. 
D. A. Kopriva And G. J. Gassner (2014), An energy stable discontinuous Galerkin spectral element discretization for variable coefficient advection problems, SIAM J. Sci. Comput., 36, pp. A2076A2099, https://doi.org/10.1137/130928650.

H.-O. Kreiss (1970), Initial boundary value problems for hyperbolic systems, Comm. Pure Appl. Math., 23, pp. 277-298.

H.-O. KReISs AND J. LoREnz (1989), Initial Boundary Value Problems and the Navier-Stokes Equations, John Wiley \& Sons, New York.

H.-O. KREISS AND G. SCHERER (1974), Finite element and finite difference methods for hyperbolic partial differential equations, in Mathematical Aspects of Finite Elements in Partial Differential Equations, Academic Press, New York, pp. 195-212.

H.-O. Kreiss And G. Scherer (1977), On the Existence of Energy Estimates for Difference Approximations for Hyperbolic Systems, Technical report, Department of Scientific Computing, Uppsala University.

F. Lauren and J. Nordström (2018), Practical inlet boundary conditions for internal flow calculations, Comput. \& Fluids, 175, pp. 159-166.

J.-P. LOHÉAC (1991), An artificial boundary condition for an advection-diffusion equation, Math. Methods Appl. Sci., 14, pp. 155-175.

M. Marden (1949), The Geometry of the Zeros of a Polynomial in a Complex Variable, American Mathematical Society, New York.

D. Michelson (1985), Initial-Boundary Value Problems for Incomplete Perturbations of Hyperbolic Systems, Lecture Notes in Appl. Math. 22, Springer-Verlag, New York.

J. NoRDSTRÖM (1989), The influence of open boundary conditions on the convergence to steady state for the Navier-Stokes equations, J. Comput. Phys., 85, pp. 210-244.

J. Nordström (2017), A roadmap to well posed and stable problems in computational physics, J. Sci. Comput., 71, pp. 365-385.

J. Nordström, S. Eriksson, And P. Eliasson (2012), Weak and strong wall boundary procedures and convergence to steady-state of the Navier-Stokes equations, J. Comput. Phys., 231, pp. 48674884 .

J. Nordström, K. Forsberg, C. Adamsson, And P. Eliasson (2003), Finite volume methods, unstructured meshes and strict stability for hyperbolic problems, Appl. Numer. Math., 45, pp. 453473.

J. Nordström, J. Gong, E. Van der Weide, And M. Svärd (2009), A stable and conservative high order multi-block method for the compressible Navier-Stokes equations, J. Comput. Phys., 228, pp. 9020-9035.

J. NoRdström AND R. GuStafsson (2003), High order finite difference approximations of electromagnetic wave propagation close to material discontinuities, J. Sci. Comput., 18, pp. 215-234.

J. Nordström, K. Mattsson, And C. Swanson (2007), Boundary conditions for a divergence free velocity-pressure formulation of the Navier-Stokes equations, J. Comput. Phys., 225, pp. 874890.

J. Nordström And M. SvÄrd (2005), Well-posed boundary conditions for the Navier-Stokes equations, SIAM J. Numer. Anal., 43, pp. 1231-1255.

H. Ranocha, P. ÖFFner, And T. Sonar (2016), Summation-by-parts operators for correction procedure via reconstruction, J. Comput. Phys., 311, pp. 299-328.

R. Sакамото (1982), Hyperbolic Boundary Value Problems, Cambridge University Press, Cambridge, UK.

J. C. STRIKWERDA (1977), Initial boundary value problems for incompletely parabolic systems, Commun. Pure Appl. Math., 30, pp. 797-822.

M. Svärd, M. H. CARPenter, And J. Nordström (2007), A stable high-order finite difference scheme for the compressible Navier-Stokes equations, far-field boundary conditions, J. Comput. Phys., 225, pp. 1020-1038.

M. SvÄrd AND J. NORDSTRÖM (2008), A stable high-order finite difference scheme for the compressible Navier-Stokes equations: No-slip wall boundary conditions, J. Comput. Phys., 227, pp. $4805-4824$.

M. SväRD AND J. NoRDSTRÖM (2014), Review of summation-by-parts schemes for initial-boundaryvalue problems, J. Comput. Phys., 268, pp. 17-38.

Z.-G. Yan, Y. Pan, G. Castiglioni, K. Hillewaert, J. Peiró, D. Moxey, and S. J. Sherwin (2020), Nektar++: Design and implementation of an implicit, spectral/hp element, compressible flow solver using a Jacobian-free Newton Krylov approach, Comput. Math. Appl., to appear.

Copyright (c) by SIAM. Unauthorized reproduction of this article is prohibited. 